\title{
A Novel Method of Equivalent Replacement Beams for Displacement Computation of Euler-Bernoulli beams
}

\author{
Zhenchao, SU ${ }^{1, a}$, Yanxia, XUE ${ }^{1, b}$ \\ ${ }^{1}$ Department of Civil Engineering, Tan Kah Kee College, Xiamen University, 363105, Zhangzhou \\ city, Fujian Province, China \\ a suzc888@126.com, ${ }^{b}$ xueyx888@126.com
}

\begin{abstract}
Keywords: method of equivalent replacement beams, superposition method, deformation computation, Euler-Bernoulli beams, mechanics of materials.

Abstract. Based on the superposition method for computing deflections of beams, the method of equivalent replacement beams is proposed for calculating displacements of beams. This method can be used to investigate the problems of deformation of statically determinate beams and indeterminate beams. This method is of clear concepts of mechanics, and the computation procedure is easy to be understood, accepted and used. An illustrative example given in this paper reveals that this method can be used to solve complicit deformation computation of beams efficiently.
\end{abstract}

\section{Introduction}

A beam is a structural element that primarily resists loads applied laterally to the beam's axis. Its mode of deflection is primarily by bending. The total effect of all the forces acting on the beam is to produce shear forces and bending moments within the beam, that in turn induce internal stresses, strains and deflections of the beam. Beams are traditionally descriptions of building or civil engineering, structural elements, but any structures such as machine frames, and other mechanical or structural systems contain beam structures are analyzed in a similar fashion.

The Bernoulli beam is named after Jacob Bernoulli, who made the most significant discoveries. Leonhard Euler and Daniel Bernoulli were the first to put together a useful theory circa $1750^{[1]}$. Euler-Bernoulli beam theory is a simplification of the linear theory of elasticity which provides a means of calculating the load-carrying and deflection characteristics of beams ${ }^{[2]}$. In engineering, deflection is the degree to which a structural element is displaced under a load. It may refer to an angle or a distance. It is well known that the calculation of deflections is an important part of structural analysis and design. For example, finding deflections is an essential ingredient in the analysis of statically indeterminate structures. Deflections are also important in dynamic analysis, as when investigating the vibrations of the aircraft or the response of buildings to earthquakes. Deflections are sometimes calculated in order to verify that they are within tolerable limits. For instance, specifications for the design of buildings usually place upper limits on the deflections. Large deflections in buildings are unsightly (and even unnerving) and can cause cracks in ceilings and walls. ${ }^{[3]}$

The deflection of beam elements is usually calculated on the basis of the Euler-Bernoulli beam equation under the following assumptions, (1) the beam is originally straight, and any taper is slight, (2) the beam experiences only linear elastic deformation, (3) the beam is slender (its length to height ratio is greater than 10), (4) only small deflections are considered (max deflection less than 1/10 the span). Deflection can be calculated by the method of integration of the curvature equation, or by methods such as, superposition method, conjugate beam method, moment-area method, virtual work method, Castigliano's method, Macaulay's method, finite difference method ${ }^{[3-6]}$, initial parameter method $^{[7]}$, etc.

In this paper, we will gain an insight into the application of the method of superposition to investigate the deflection of Euler-Bernoulli beams, and propose the concept of equivalent replacement beam(ERB) and use the method of equivalent replacement beam(MERB) to some examples for computing deflections of various beams and rigid frames. The article is organized as 
follows. In Section 2, the concept of ERB is established, and a corresponding proposition will be proposed. In Section 3, an illustrative example of using MERB to calculate deflections of statically beans will be given to demonstrate MERB's effectiveness.

\section{Theoretical fundament of MERB}

A cantilever beam is a kind of typical and basic statically determinate beam, and its deflections, such as the deflection and angle of rotation at the free end, have been discussed in many current textbooks ${ }^{[4-11]}$ of mechanics of materials.

For example, the deflection and angle of rotation of cantilever beam $A B$ acted in a couple $M_{\mathrm{e}}$ at free end $B$ shown in Fig.1 are

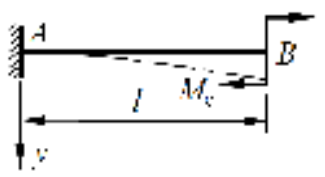

Fig.1 Cantilever beam acted with a couple

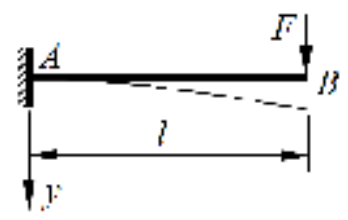

Fig.2 Cantilever beam acted with a concentrated force

$$
\theta_{B}=\frac{M_{\mathrm{e}} l}{E I}(\mathrm{P}), \quad w_{B}=\frac{M_{\mathrm{e}} l^{2}}{2 E I}(\$)
$$

in which, $w_{B}$ and $\theta_{B}$ are the deflection and angle of rotation of the beam at the end $B, E I$ is the flexural rigidity of the beam and $l$ is its length.

The deflection and angle of rotation of cantilever beam $A B$ acted with a concentrated force $F$ at free end $B$ shown in Fig. 2 are

$$
\theta_{B}=\frac{F l^{2}}{2 E I}(\mathrm{P}), \quad w_{B}=\frac{F l^{3}}{3 E I}(\$)
$$

The deflection and angle of rotation of cantilever beam $A B$ acted with a uniform distributed load of intensity $q$ throughout the span of the beam shown in Fig.3 are

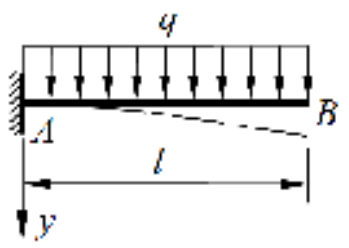

Fig.3 Cantilever beam acted with uniform load

$$
\theta_{B}=\frac{q l^{3}}{6 E I}(\mathrm{P}), \quad w_{B}=\frac{q l^{4}}{8 E I}(\$)
$$

These results are essential and important for engineering practice. At the same time, they play a fundamental role in the course of structural mechanics or engineering structure. In this section, based on the above results, we will try to use cantilever beams to replace other type of beams to analyze the deflections of various types of beams.

First, the concept of ERB here is defined as follows.

An ERB means that a cantilever beam can be regarded as a substitute of another type of beam in the sense of having the equivalent (inner and outer) forces and equivalent deflection at all sections over the total beam in addition to same material and cross section shape.

Proposition: 
For a straight statically determinate beam with constant section, its ERB must exist as long as the deflection and angle of rotation of ERB at the fixed end equals to its origin, and the actions on ERB are the same as its origin.

For example, the simple supported beam $A B$ acted with uniform load of intensity $q$ shown in Fig.4, its ERB $A_{1} B_{1}$ can be constructed shown in Fig.5, in which $\theta_{A}$ and $F_{B}$ respectively stand for the angle of rotation of end $A$ and reaction at end $B$ of beam $A B$. Namely, the beam $A_{1} B_{1}$ in Fig.5 has the same deformation and the position as beam $A B$ in Fig.4.

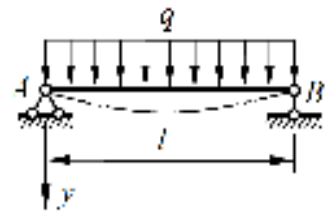

Fig.4 Simple supported beam $A B$ acted with uniform load

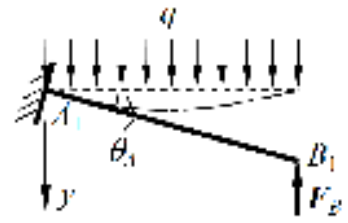

Fig.5 The ERB $A_{1} B_{1}$ for the beam $A B$ shown in Fig.4

For a definite statically determinate beam, its deformation is decided by the actions (active and passive actions, including forces and couples) and boundary conditions. Obviously, the beam $A_{1} B_{1}$ in Fig.5 has the same actions, deflection and angle of rotation at left end as beam $A B$ in Fig.4. Obviously, ERB of a beam is not unique. For example, another ERB of the beam $A B$ shown in Fig. 4 can be built by fixing the end $B$ and releasing the end $A$. This non-uniqueness provides people some flexibilities to choose a simple way to the solution.

The theoretical basis of the proposition is the superposition method under the assumption of small deformation. The proof can be simply accomplished with the initial parameter method ${ }^{[7]}$.

\section{Computing deflections of statically determinate beam with MERB}

Example. A simple beam ACB supports a concentrated load F acting at the position shown in Fig.6. Determine the angles of rotation at supports $\mathrm{A}$ and $\mathrm{B}$, and the deflection of the midpoint of the beam under load F.ection of midpoint of the beam under load $F .(a \geq b)$

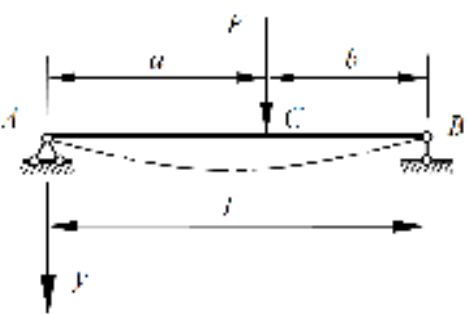

Fig.6 Simple beam with a concentrated load

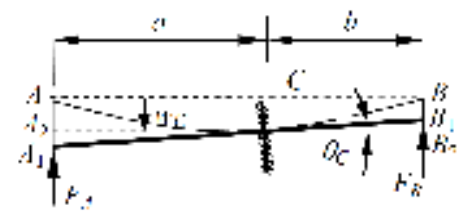

Fig.7 The ERB of beam $A B$ in Fig.6

Solution. By equilibrium law, the reactive forces acting at supports $A$ and $B$ of the beam $A B$ in Fig.6 are

$$
F_{A}=\frac{b}{l} F, \quad F_{B}=\frac{a}{l} F
$$

To use the results of (2), assuming that the deflection and the angle of rotation at point $C$ indicated by $w_{C}$ and $\theta_{C}$ shown in Fig. 6 are small quantity, by fixing the cross section $C$, the ERB of beam $A B$ in Fig. 6 can be constructed with $A_{1} C B_{1}$ as shown in Fig.7, in which the forces of $F_{A}$ and $F_{B}$ are the reactive forces at the ends $A$ and $B$ in Fig.6, and the force $F$ acting at the point $C$ can be neglected because that the cross section $C$ is fixed.

By the geometric relations in Fig.7, one reads 
$\overline{A A_{1}}=\overline{A A_{2}}+\overline{A_{2} A_{1}}, \overline{B B_{1}}=\overline{B B_{2}}-\overline{B_{2} B_{1}}$

Substituting the results (2) into the above relations, one reads the equations as follows.

$$
w_{C}+\theta_{C} \cdot a=\frac{F_{A} \cdot a^{3}}{3 E I}=\frac{F b \cdot a^{3}}{3 E I l}, w_{C}-\theta_{C} \cdot b=\frac{F_{B} \cdot b^{3}}{3 E I}=\frac{F a \cdot b^{3}}{3 E I l}
$$

The unknowns $w_{C}$ and $\theta_{C}$ can be found out respectively from the equations (4)

$$
w_{C}=\frac{F a^{2} b^{2}}{3 E I l}, \theta_{C}=\frac{F a b(a-b)}{3 E I l}
$$

Based on the Fig.7 and (5), the angles of rotation at ends $A$ and $B$ respectively are

$$
\begin{aligned}
& \theta_{A}=\frac{F_{A} a^{2}}{2 E I}-\theta_{C}=\frac{F a^{2} b}{2 E I l}-\frac{F a b(a-b)}{3 E I l}=\frac{F a b(l+b)}{6 E I l}(\mathrm{P}) \\
& \theta_{B}=\frac{F_{B} b^{2}}{2 E I}+\theta_{C}=\frac{F a b^{2}}{2 E I l}+\frac{F a b(a-b)}{3 E I l}=\frac{F a b(l+a)}{6 E I l}(\mathrm{Q})
\end{aligned}
$$

Since $a \geq b$, the midpoint of the beam is at the left part, employing the results (2), (5) and the method of superposition, the deflection of the midpoint of the beam is

$$
w\left(\frac{l}{2}\right)=w_{C}+\theta_{C} \cdot\left(a-\frac{l}{2}\right)-\frac{F_{A}\left(a-\frac{l}{2}\right)^{3}}{3 E I}-\frac{F_{A}\left(\frac{l}{2}\right) \cdot\left(a-\frac{l}{2}\right)^{2}}{2 E I}=\frac{F b\left(3 l^{2}-4 b^{2}\right)}{48 E I}(\downarrow)
$$

Note, the above procedure is rather simpler than the traditional method, as an example in [3].

\section{Conclusions}

Illustrative Example given in this paper reveals that MERB has strong simplicity, validity and applicability for solving the problems of deformations of Euler-Bernoulli beams. MERB can give a clear clue to find deflections of beams, and has distinct concepts of mechanics. MERB give students and researchers a useful tool to investigate the deformations of beams. Meanwhile, MERB can be used for statically indeterminate beams and rigid frames for investigating its deformations. The example given in this paper demonstrates that this method is very easy to understand, accept and use. Whereas, MERB is based on the method of superposition. Thus, MERB is only suitable for linear beams or rigid frames with constant cross section.

\section{References}

[1] C. Truesdell, Outline of the History of Flexible or Elastic Bodies to 1788. The Journal of the Acoustical Society of America 32, 1647-56 (1960). doi: 10.1121/1.1907980.

[2] Timoshenko, S., "History of strength of materials", McGraw-Hill, New York, 1953.

[3] James M. Gere, "Mechanics of Materials", Fifth Ed., Brooks/Cole Publishing Company, Thomson Learning, 2001.

[4] S. Timoshenko, J. Gere, "Mechanics of Materials", van Nordstrand Reinhold Company, New York, 1972.

[5] R. C. Hibbeler. "Mechanics of Materials", Fifth Edition. Pearson Education, Prentice Hall. 2003.

[6] Andrew Pytel, Jaan Kiusalaas. "Mechanics of Materials", second Ed., CENGAGE Learning, 2010.

[7] Sun Xunfang, Fang Xiaoshu, Guan Laitai, "Mechanics of Materials ( I )" (in Chinese), 5ed., Higher Education Press, Beijing, 2009. 